\title{
Flash microbiocide: A Rapid and Economic Method for Determination of MBC and MFC
}

\author{
Camila Hernandes*, Juliana da Silva Coppede, Bianca Waléria Bertoni, Suzelei de Castro França, \\ Ana Maria Soares Pereira
}

Department of Biotechnology, University of Ribeirão Preto, Ribeirão Preto, Brazil.

Email: *camila.hernandes@hotmail.com

Received February $24^{\text {th }}, 2013$; revised March $28^{\text {th }}, 2013$; accepted April $4^{\text {th }}, 2013$

Copyright (C) 2013 Camila Hernandes et al. This is an open access article distributed under the Creative Commons Attribution License, which permits unrestricted use, distribution, and reproduction in any medium, provided the original work is properly cited.

\begin{abstract}
Although nowadays there are methods for determining the Minimal Bactericidal Concentration and Minimal Fungicidal Concentration, it is indispensable that the establishment of innovative methodologies could be more practical and cheaper. The new methodology Flash microbiocide is an assay in which one aliquot from 96 well plate of Minimal Inhibitory Concentration test is transferred to another plate containing different culture medium. The correspondence with the reference methods described in the National Committee for Clinical Laboratory Standards (NCCLS-CLSI) document M26-A was achieved, denoting the efficiency of this fast and simple method.
\end{abstract}

Keywords: Antimicrobial Activity; Minimal Inhibitory Concentration; Minimal Bactericidal Concentration; Minimal Fungicidal Concentration; Methodology

\section{Introduction}

The determination of Minimal Inhibitory Concentration (MIC) is sufficient to indicate the ability of a compound to inhibit microbial replication. However, for patients with immunosuppressive infections or inflammatory diseases, such as osteomyelitis, it is necessary to determine the Minimal Bactericidal Concentration (MBC) and Minimal Fungicidal Concentration (MFC) to accurately determine the dosage of the antimicrobial agent to be prescribed, which will contribute to the success of the treatment [1-3].

Although there are numerous antibiotics available in the market, with defined ranges of MIC and MBC, the tolerance and resistance shown by microorganisms demands the development of new antimicrobial agents as well as new methodologies to precisely quantify the microbicidal activity of the new pharmaceuticals $[3,4]$.

Nowadays, the techniques for determining bactericidal or bacteriostatic action include MBC, time-kill assay and serum bactericidal titer (SBT). However, those techniques present problems related to the interpretation of results and reproducibility [5], moreover they are expensive and require a long execution time. Thus, it is necessary that the establishment and standardization of new

"Corresponding author. and low-cost methods are capable to overcome the limitations of the existing techniques.

The aim of this work is to establish a new method for determining MBC and MFC which is rapid, easily interpreted, reproducible and inexpensive.

\section{Materials and Methods}

\subsection{Actives}

Ampicillin (Sigma Aldrich $\left.{ }^{\circledR}\right)$, Gentamycin sulphate (Ourofino $\left.{ }^{\circledR}\right)$, Amphotericin B (Sigma Aldrich ${ }^{\circledR}$ ), lyophilized aqueous extract of stem bark of Stryphnodendron adstringens (barbatimão), and Lippia velutina essential oil, with the latter being a non-commercial active extracted at the Biotechnology Unit of the University of Ribeirao Preto.

\subsection{Preparation of the actives}

Ampicillin $\left(64 \mu \mathrm{g} \cdot \mathrm{mL}^{-1}\right)$, Gentamycin sulphate $\left(64 \mu \mathrm{g} \cdot \mathrm{mL}^{-1}\right)$, Amphotericin B $\left(32 \mu \mathrm{g} \cdot \mathrm{mL}^{-1}\right)$, S. adstringens extract $(2$ $\left.\mathrm{mg} \cdot \mathrm{mL}^{-1}\right)$, L. velutina essential oil $\left(40 \mu \mathrm{L} \cdot \mathrm{mL}^{-1}\right.$ dissolved into 10\% Tween 80 (Dinâmica)).

\subsection{Microorganisms}

Bacillus subtilis ATCC 6633, Escherichia coli ATCC 
25922, Pseudomonas aeruginosa ATCC 9027, Staphylococcus aureus ATCC 6538, Candida krusei ATCC 6258 and Aspergillus niger ATCC 16404.

\subsection{Antimicrobial Susceptibility Testing}

Firstly, the Minimal Inhibitory Concentration (MIC) was determined by microdilution method following CLSI guidelines [6-8].

\subsection{Determination of Bactericidal and Fungicidal Activity}

The Minimal Bactericidal Concentration and Minimal Fungicidal Concentration were determined following CLSI guidelines [3] with the objective to compare the results obtained using the new method.

\subsection{New Method for Determining Bactericidal and Fungicidal Activity: Methodology "Flash"}

The new method for determining MBC and MFC, was carried out with material collected from 96 well plate used for establishing the MIC of agents. Aliquots of 10 and $100 \mu \mathrm{L}$ were transferred to well plates containing different culture media (Mueller Hinton for bacterial strains and RPMI for fungal strains). The plates were then incubated for 24 or 48 hours according to requirements for each strain. Bactericidal and fungicidal activities having minimum concentration showed no microorganism growth. Data were compared with those from assays based on CLSI [3] (Figure 1).

\section{Results}

With regard to the bacterial strains, $100 \%$ reproducibility was observed for E. coli and P. aeruginosa, whereas differences in MBC and MFC were found for S. aureus and C. krusei, despite were not significant (1 well only) (Table 1). For the strain B. subtillis, data obtained for MBC (CLSI method) and the Flash microbiocide method were equivalent, although the MIC was inferior. When A. niger was used, both methods were not found to be efficient as they showed reproducibility.

Since obtained data indicated that there is no difference in reproducibility when using 10 or $100 \mu \mathrm{L}$ aliquots (data not shown), the aliquot of $10 \mu \mathrm{L}$ was standardized for containing minimal concentration of antibiotic to be transferred to the new culture medium.

\section{Discussion}

Obtained results were similar to those reported in the protocol endorsed by CLSI [3], ensuring the use of the Flash microbiocide method as an effective technique for determining microbicidal activity.
The main advantage of this innovative method is that it was standardized for determining not only the MBC and MFC of commercial substances but also of extracts and essential oils of plants. Additionally, it reduces runtime testing and decreases costs by $60 \%$ if compared to the M26-A methodology recommended by the CLSI.

Another improvement achieved by Flash microbiocide is the use of small-volume aliquots $(10 \mu \mathrm{L})$ transferred to fresh culture medium, reducing the chances of antimicrobial agents influence the results, considering that different authors have reported that the permanence of the antimicrobial agents existing in the aliquots transferred to the culture medium interferes with the determination of MBC and MFC $[5,9,10]$.

The already mentioned advantages permit to affirm that using the Flash microbiocide method it is possible to safely evaluate the minimum bactericidal concentration and the minimum fungicidal concentration of actives to be used in the development of innovative antimicrobial drugs. Moreover, the methodology may be introduced in the laboratory routine associated with antibiograms, to help in the treatment of and immunocompromised patients infected with microorganisms resistant to common therapy.

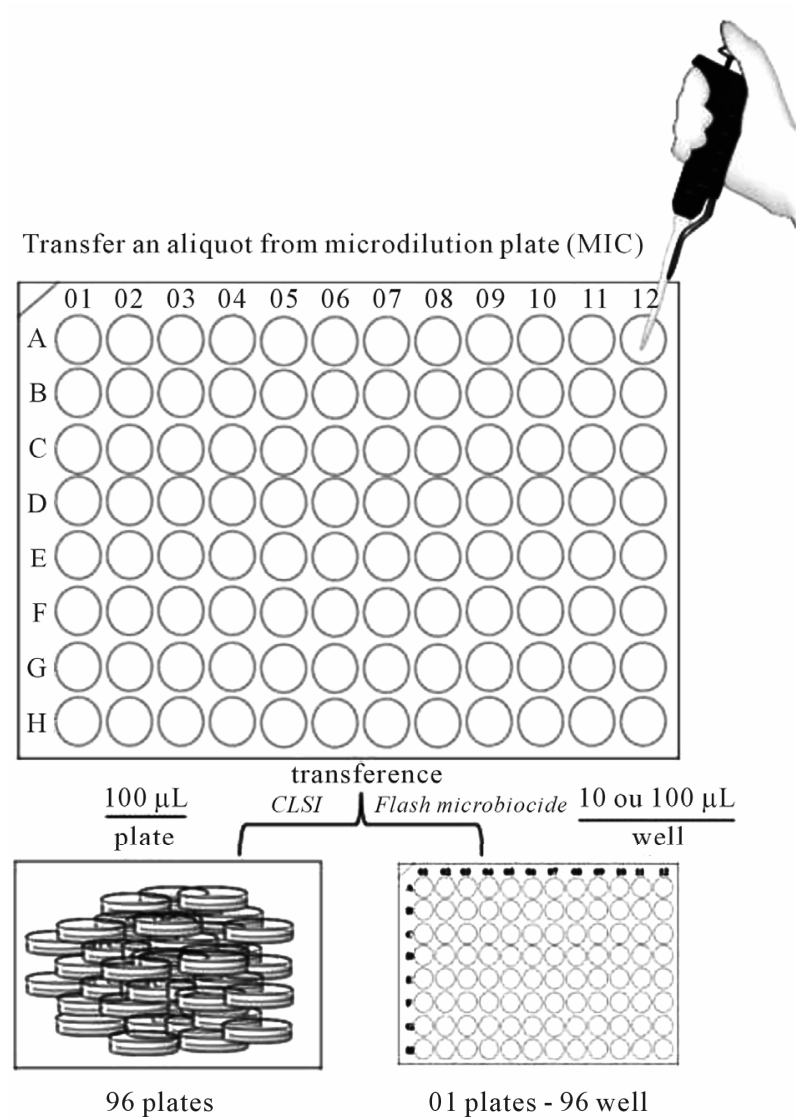

Figure 1. Scheme representing the assay procedures to evaluate the MBC and MFC of actives. 
Table 1. Evaluation of antimicrobial activity of antibiotics and vegetal actives by preconized method CLSI and with the new methodology Flash microbiocide.

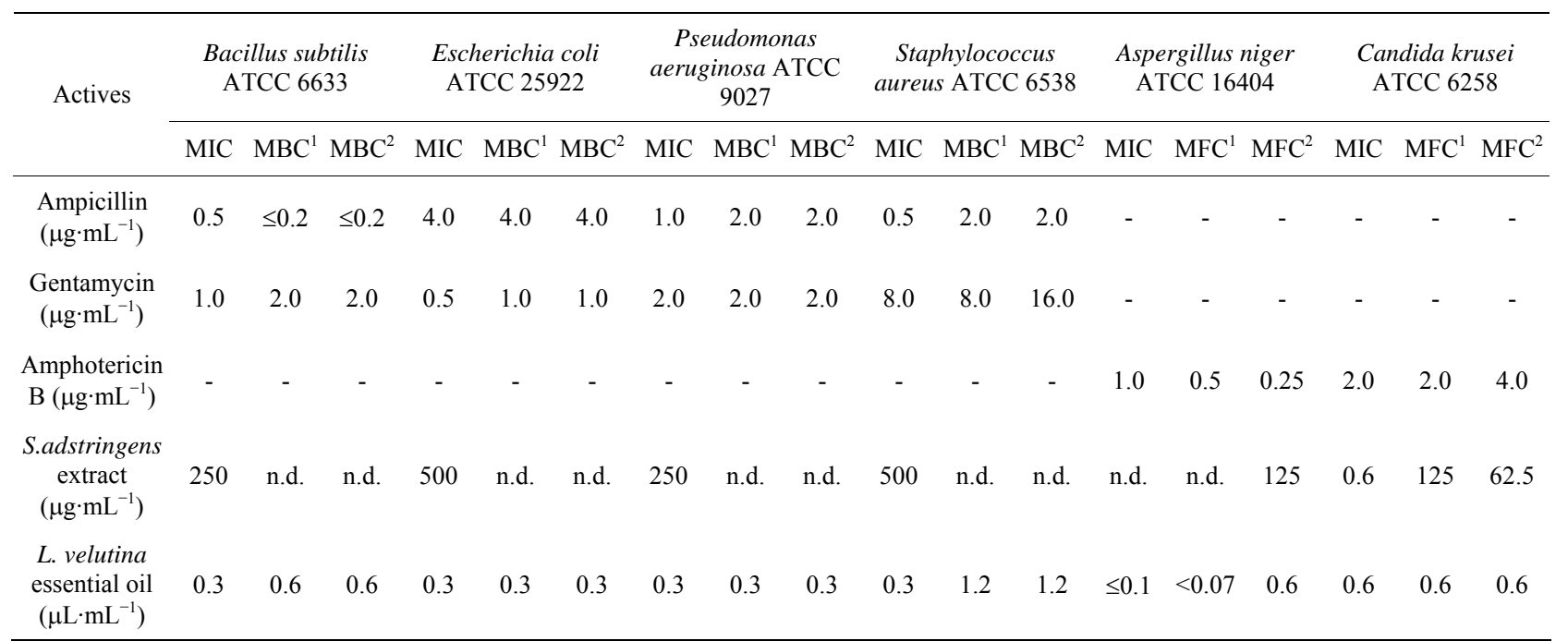

$\overline{\mathrm{MIC}}=$ minimal inhibitory concentration; $\mathrm{MBC}^{1}=$ minimal bactericidal concentration $(\mathrm{CLSI}) ; \mathrm{MBC}^{2}=$ minimal bactericidal concentration $($ Flash $)$; $\mathrm{MFC}^{1}=$ minimal fungicidal concentration (CLSI); $\mathrm{MFC}^{2}=$ minimal fungicidal concentration (Flash microbiocide); ( $)$ : not assayed; (n.d): not determined (>1000 $\left.\mu \mathrm{g} \cdot \mathrm{mL}^{-1}\right)$; assays $\mathrm{MBC}^{2}$ and $\mathrm{MFC}^{2}$ were performed with aliquotes of $10 \mu \mathrm{L}$ from MIC.

\section{Acknowledgements}

This work was supported by University of Ribeirão Preto (UNAERP), FAPESP 2010/07970-7 and Ourofino.

\section{REFERENCES}

[1] W. M. Scheld and M. A. Sande, "Bactericidal versus Bacteriostatic Antibiotic Therapy of Experimental Pneumococcal Meningitis in Rabbits," Journal of Clinical Investigation, Vol. 71, No. 3, 1983, pp. 411-419. doi:10.1172/JCI110785

[2] J. P. Sculier and J. Klastersky, "Significance of Serum Bactericidal Activity in Gram-Negative Bacillary Bacteremia in Patients with and without Granulocytopenia," American Journal of Medicine, Vol. 76, No. 3, 1984, pp. 429-435. doi:10.1016/0002-9343(84)90662-4

[3] Clinical and Laboratory Institute, "Methods for Determining Bactericidal Activity of Antimicrobial Agents," Approved Guideline, CLSI Document M26-A, Wayne, 1999.

[4] S. Handwerger and A. Tomaz, "Antibiotic Tolerance among Clinical Isolates of Bacteria," Reviews of Infectious Diseases, Vol. 7, No. 3, 1985, pp. 368-386. doi:10.1093/clinids/7.3.368

[5] G. A. Pankey and L. D. Sabath, "Clinical Relevance of
Bacteriostatic versus Bactericidal Mechanisms of Action in the Treatment of Gram Positive Bacterial Infections," Clinical Infectious Diseases, Vol. 38, No. 6, 2004, pp. 864-870. doi:10.1086/381972

[6] Clinical and Laboratory Institute, "Methods for Dilution Antimicrobial Susceptibility Tests for Bacteria That Grow Aerobically," 6th Edition, CLSI Document M7-A6, Wayne, 2003.

[7] Clinical and Laboratory Institute, "Reference Method for Broth Dilution Antifungal Susceptibility Testing of Yeasts," 3rd Edition, CLSI Document M27-A2, Wayne, 2002.

[8] Clinical and Laboratory Institute, "Reference Method for Broth Dilution Antifungal Susceptibility Testing of Filamentous Fungi," 2nd Edition, CLSI Document M38-A, Wayne, 2008.

[9] A. L. Barry and R. A. Lasner, "In Vitro Methods for Determining Minimum Lethal Concentrations of Antibacterial Agents," American Journal of Clinical Pathology, Vol. 71, No. 1, 1979, pp. 88-92.

[10] J. Dankert, Y. Holloway, W. Joldersma and J. Hess, "Important of Minimizing Carry over Effect at Subculture in the Detection of Penicillin Tolerant Viridans Group Streptococci," Antimicrobial Agents and Chemotherapy, Vol. 23, No. 4, 1983, pp. 232-235. doi:10.1128/AAC.23.4.614 